
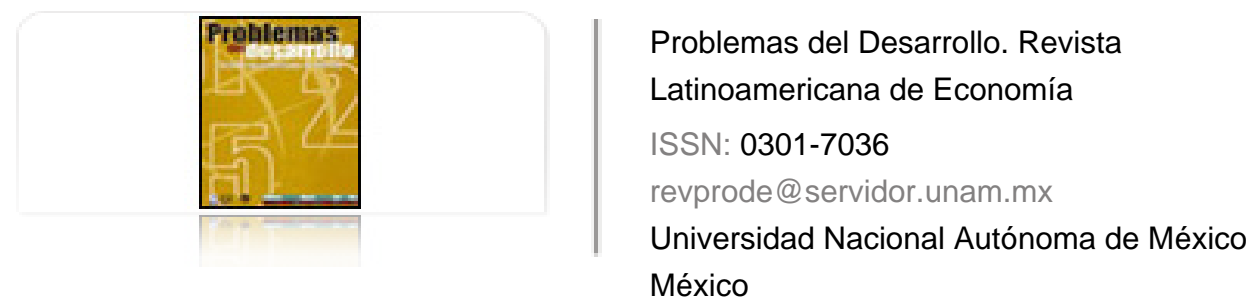

Mochi Alemán, Prudencio

La industria del software en México

Problemas del Desarrollo. Revista Latinoamericana de Economía, vol. 35, núm. 137, 2004, pp. 41-58

Universidad Nacional Autónoma de México

Distrito Federal, México

Disponible en: http://www.redalyc.org/articulo.oa?id=11825947003

- Cómo citar el artículo

Número completo

- Más información del artículo

Página de la revista en redalyc.org

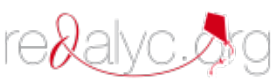

Sistema de Información Científica

Red de Revistas Científicas de América Latina, el Caribe, España y Portugal Proyecto académico sin fines de lucro, desarrollado bajo la iniciativa de acceso abierto 


\title{
LA INDUSTRIA DEL SOFTWARE EN MÉXICO
}

\section{Prudencio Mochi Alemán**}

Fecha de recepción: 29 de abril de 2004. Fecha de aceptación: 11 de junio de 2004.

\section{Resúmenes}

El trabajo que presentamos tiene como objetivo central el estudio - en los contextos internacional y latinoamericano- de la industria del software en México. Ésta es la de más alto crecimiento y de mayor significación de las SE-I (sector electrónico informático) en el ámbito mundial. Se tomaron como casos de referencia investigaciones realizadas en India, Irlanda e Israel, así como en Brasil, Argentina, Uruguay y Costa Rica, por ser países en los cuales a partir de la división global del trabajo — desde la década de los ochenta - destacan por su producción en software y por su inserción en otras naciones. Para analizar el caso de México se recuperaron los datos generales del subsector y se seleccionaron 30 empresas nacionales e internacionales que desarrollan esos productos a la medida, por considerar que son las más dinámicas e innovadoras de esta industria. El proyecto en su conjunto abarca el análisis de experiencias citadas, con más énfasis en los casos latinoamericanos, por las posibilidades de acceso a la información más inmediata y, sobre todo, la situación particular de México.

Palabras clave: software, inserción, países, industrialización tardía.

\begin{abstract}
The study centers on the software industry in Mexico - in the international and Latin American contexts. This has the highest growth and the greatest significance for the IES (informatics electronics sector) worldwide. Studies done in India, Ireland and Israel, as well as Brazil, Argentina, Uruguay and Costa Rica were used as reference cases, because these are countries involved in the global division of labor since the 1980s — and which stand out for their software production and insertion in other countries. To analyze the case of Mexico, general data were compiled for the sub-sector and a selection made of 30 national and international companies considered to be the most dynamic and innovative in this industry in developing these custom-designed products.
\end{abstract}

Key terms: software, insertion, countries, late industrialization, software industry, informatics electronics sector.

* Investigador de Centro Regional de Investigaciones Multidisciplinarias (CRIM-UNAM). Correo electrónico: mochiprudencio@hotmail.com 
Résumé

Le travail que nous présentons a, comme objectif central, l'étude —dans les contextes international et latino-américain - de l'industrie du logiciel au Mexique. C'est l'industrie dont la croissance a été la plus élevée et dont la signification des SEI (secteur électronique informatique) dans le domaine mondial a été le plus grand. Cette étude est basée sur des investigations, comme des cas de référence, réalisées en Inde, en Irlande et en Israël, de même qu'au Brésil, en Argentine, en Uruguay et au Costa Rica, qui sont des pays qui se distinguent, à partir de la division globale du travail depuis les années 80, par leur production en software et par leur insertion dans d'autres nations. Pour l'analyse du cas du Mexique, on a récupéré les données générales du sous secteur et on a sélectionné 30 entreprises nationales et internationales qui développent ces produits sur mesure, que l'on a considéré comme les plus dynamiques et les plus innovatrices.

Mots clés: software, insertion, pays, industrialisation tardive, industrie du software, secteur électronique informatique.

\section{Resumo}

O principal objetivo deste trabalho é o estudo da indústria do software no México, dentro dos contextos internacional e latino-americano. No âmbito do SEI (Setor Eletrônico Informático) a indústria de desenvolvimento de software é a de mais alto crescimento e de maior importância em escala mundial. Foram utilizadas como referência, pesquisas realizadas na Índia, Irlanda e Israel, assim como no Brasil, Argentina, Uruguai e Costa Rica, devido à posição de destaque que esses países alcançaram — com a divisão global do trabalho a partir da década dos oitentapela alta produção de softwares e pela conseqüente disseminação dos mesmos para outras nações. Para analisar o caso do México, foram recuperados dados gerais do setor e selecionadas, para estudo, 30 empresas nacionais e internacionais, desenvolvedoras de produtos sob medida e consideradas as mais dinâmicas e inovadoras desta indústria.

Palavras-chave: software, inserção, países, industrialização tardia, indústria do software, setor eletrônico informático. 


\section{Introducción}

$\mathrm{L}$

os avances tecnológicos experimentados durante las últimas décadas del siglo $\mathrm{xx}$ impulsaron una serie de procesos definidos como una nueva revolución productiva o industrial. Tal fenómeno está relacionado con el surgimiento de una etapa del sistema de producción capitalista caracterizada por la importancia creciente de la innovación tecnológica y del conocimiento en la generación de valor, en un contexto de globalización económica (Dabat, 2002).

En este escenario, las tecnologías de la información y la comunicación (TIC) han adquirido gran importancia debido a factores como la expansión acelerada y los cambios revolucionarios en el sistema de telecomunicaciones, los procesos vinculados con el desarrollo de Internet, la introducción y demanda exponencial de las computadoras personales, así como de programas de cómputo especializados.

Tales elementos se encuentran asociados al desarrollo y uso creciente de una tecnología multifuncional, el software, cuyas características dificultan su definición: es un elemento dual, a la vez servicio y producto, intangible y necesario para muchas actividades; desempeña un papel clave en la reconfiguración de las industrias, indispensable para el procesamiento de datos y el funcionamiento de los equipos de hardware; se le incorpora cada vez más a diversos productos industriales de uso cotidiano. Su gran dinamismo económico propicia el desarrollo de nuevas áreas y crea nuevas oportunidades de empleo. Ha generado una industria importante, cuyos campos fundamentales son la ingeniería del software y los servicios informáticos (ISSI), que tienen una estructura compleja y gran capacidad de innovación.

En este contexto se desarrolla el presente trabajo sobre la industria del software, de más alto crecimiento y de mayor significación dentro de las SE-I (sector electrónico-informático) en el ámbito internacional. Su papel en la dinámica de las economías modernas es cada vez más importante, ya que su expansión está estrechamente asociada a la introducción voluminosa de las nuevas tecnologías de la información y las comunicaciones, las cuales están redefiniendo de manera acelerada las formas de producir, vender y competir en prácticamente todos los sectores productores de bienes y servicios. Se trata, en consecuencia, de una industria estratégica en el actual contexto mundial y cuyo desarrollo se impulsa por diversas vías en Estados Unidos, así como en varias naciones de industrialización reciente, tales como India, Irlanda e Israel, los cuales a partir del desarrollo de la división global del trabajo, desde la década de los ochenta, han comenzado su inserción en el mercado internacional. 
En América Latina, la industria también sigue un proceso de maduración incipiente que se manifiesta en su crecimiento casi ininterrumpido durante los años recientes. Además, en toda la región se están emitiendo políticas gubernamentales activas que estimulan la creación de empresas, el desarrollo de las ya existentes y la promoción de tecnología e infraestructura en telecomunicaciones que los países requieren para sentar las bases de su crecimiento a largo plazo.

Las oportunidades y retos que plantea consolidar la industria del software dejan clara la necesidad y urgencia de convocar a todos los sectores para construir una visión colectiva, que facilite su inserción en la economía internacional y el desarrollo de los demás ámbitos de las economías nacionales.

Cabe destacar un hecho sorprendente. Pese a la importancia y rápido crecimiento de la industria del software en todo el mundo, es relativamente escasa la investigación académica del tema, específicamente, desde el punto de vista socioeconómico. Así, hay gran cantidad de trabajos que estudian las formas de competencias o la dinámica de la innovación en ramas como la automotriz, química, computación, pero son pocos los que lo hacen con el subsector de software. Este problema también se extiende al campo de los datos y estadísticas, en los que existen carencias notorias. De ahí la importancia de nuestra investigación, fundada en trabajos y fuentes internacionales de los cuales se extrajeron elementos para caracterizar las tendencias y características básicas del subsector.

En primer lugar se aborda la ubicación del subsector en el contexto internacional. Luego se describe el estudio de experiencias en India, Irlanda, Israel y en el contexto latinoamericano, Brasil, Argentina, Uruguay y Costa Rica. Posteriormente ofrecemos un panorama general sobre el desarrollo de la industria de software en México, que destaca por ser el más dinámico entre las tecnologías de la información y el conocimiento, con un crecimiento de $12.5 \%$ en el 2002 para software en paquete ${ }^{1}$ y de $9 \%$ para software a la medida $^{2}$ (Mochi, 2003:117). Después presentaremos algunas consideraciones finales.

Las interrogantes que pretendimos responder fueron: ¿Cuál es el grado de desarrollo de la industria internacional del software en el marco del desarrollo de las TIC? ¿Qué posibilidades tiene México de insertarse en el mercado internacional como productor de software competitivo e innovador? ¿Qué tipo de incentivos gubernamentales existen en México? ¿Qué recursos humanos preparados para esta industria hay en México? ¿Cuáles son las fortalezas y debilidades del subsector en otras realidades internacionales que compiten con México? ¿Cuáles son las fortalezas y debilidades del subsector en México? La

1 Como su nombre lo indica, el software empaquetado se ofrece en paquetes confeccionados, se dirigen al mercado masivo, de manera estándar, generalmente su uso es más fácil y viene acompañado de manuales que explican todas sus funciones.

2 El software desarrollado a la medida, en cambio, exige de acuerdo con su complejidad algún grado de personalización o adaptación a la organización en la cual serán implantados. 
idea era indagar en qué medida se podía hablar de un subsector competitivo y consolidado en el país.

El problema y los presupuestos planteados que acompañan el trabajo fueron:

a) la industria del software es de importancia estratégica central para el desarrollo futuro del país, dado su papel decisivo dentro del conjunto de SE-I, y como el subsector de más rápido crecimiento dentro del mismo;

b) la calificación de la fuerza de trabajo y el desarrollo de opciones educativas resultan fundamentales dentro de la industria del software;

c) la industria se caracteriza por el peso particular de la micro y pequeña empresa y por su carácter innovador, $\mathrm{y}$

d) para los países de industrialización reciente (por tanto, para México), se requieren políticas públicas que impulsen ese desarrollo y les permitan escalar niveles más elevados de esa actividad.

Los presupuestos particulares de la investigación fueron los siguientes:

1) México tiene un desarrollo relativamente escaso de la industria del software, a tal grado que no se han generado sistemas operativos ni lenguajes, salvo de carácter experimental y académico, y la exportación es limitada y está concentrada en pocas empresas. Esto se debe en gran parte a la falta de políticas activas, tanto industriales como de fomento exportador. Prevalecen las empresas desarrolladoras de software a la medida y las distribuidoras de software producido por terceros o por las casas matrices.

2) A pesar del crecimiento de la demanda de trabajos calificados para esta industria en México, no existe un desarrollo suficientemente amplio de la oferta educativa ni un vínculo estrecho entre academia y empresa.

3) La infraestructura disponible en materia de telecomunicaciones en México está poco desarrollada, aunque existen esfuerzos para expandirla.

Consideramos necesario, para su mejor entendimiento, aclarar algunos conceptos, los cuales describen los tipos de empresas ${ }^{3}$ y rubros $^{4}$ vinculados a esta rama industrial, así como los segmentos de productos que caracterizan al subsector.

3 Tomando en cuenta los parámetros internacionales, clasificamos las compañías en: a) empresas desarrolladoras de software; $b$ ) subcontratistas de las mismas; c) empresas de servicios (instalación de programas, configuración, pruebas, puesta a punto, elaboración de manuales, entre otros).

4 Si bien no es posible trazar una delimitación precisa entre todas las categorías en las que puede dividirse la producción de software de acuerdo con sus características, importancia y complejidad, pueden separase en cuatro grupos: 1) sistemas operativos; 2) software aplicativos o productos 


\section{La industria internacional de software}

El cambio de paradigma tecno-económico que llevó a la nueva revolución productiva industrial, tuvo como principal motor la conversión del sector electrónico-informático 5 (SE-I) en el eje central de la economía mundial (Dabat; Ordóñez, 2003).

Desde el punto de vista de la transformación económica, esta dinámica es resultado de una búsqueda de soluciones a la crisis del modelo capitalista fordista-keynesiano ${ }^{6}$ que se expresó con la fuerte caída de la tasa de ganancia de los años sesenta y setenta. Esta tendencia se manifiesta en el cambio de desarrollo de la inversión en la industria automotrizpetrolera y, contemporáneamente, en la diversificación de la vieja industria informática integrada verticalmente. A su vez implicó el paso a la nueva industria centrada en computadoras y en la red de los años ochenta y noventa. El sector informático caracterizado por empresas con procesos verticales compactos dio lugar a formas descentralizadas en distintos sectores industriales y mercados diferenciados, básicamente hardware, software, armado final, comercialización, entre otros.

Este nuevo ciclo industrial se vincula con la llamada nueva economía o economía del conocimiento $^{7}$ reflejada en el gran crecimiento del sector durante la década de los noventa. Asimismo, tendrá grandes consecuencias para la economía mundial.

En primer término tiende a modificar las relaciones económicas de fuerza entre las grandes potencias industriales a favor de Estados Unidos (por su liderazgo tecnológico y empresarial en sectores estratégicos como procesadores, software e Internet) y en contra de Japón (por su relativa débil posición en los nuevos sectores de punta), con resultados desiguales y aún inciertos para Europa Occidental (favorables en los países escandinavos e Irlanda e Inglaterra; pero relativamente preocupantes en Francia y Alemania) (Dabat; Ordóñez, 2003:51).

Otro factor por considerar son las consecuencias aún desiguales para países y regiones menos industrializados. Los de industrialización reciente o semindustrializados han logra-

empaquetados de mercado masivo; 3) soluciones empresariales o software desarrollado a la medida y servicios informáticos; 4) software embebido (es el incorporado en distintos tipos de maquinarias, equipos y dispositivos de consumo).

5 El sector electrónico-informático (SE-I) está integrado por cinco subsectores considerados por Miller (1993) y descritos en el trabajo de Dabat y Ordóñez (2003). Comprende a semiconductores, computadoras, telecomunicaciones, equipo industrial y electrónica de consumo, y como subgrupo consideran al software (paquetería y servicios de programación) y servicios a la computación (diseños de sistemas integrados de computadoras, procesamiento y preparación de datos y otros de menor importancia).

6 Entendemos por modelo fordista - keynesiano la organización económica basada en la producción y consumo de masa, estandarizada, en la organización de la gran empresa vertical, en la extensión del trabajo asalariado y el obrero masa, cuyo paradigma eran las fábricas de automotores, pero que se extendió como modelo de organización, definiendo la sociedad y el Estado.

7 El conocimiento es, dentro de la nueva revolución productiva industrial, central para el desarrollo de las actividades económicas. Según la definición de la OCDE, la producción, distribución y uso del conocimiento constituyen el motor principal del crecimiento y de la creación de riqueza y empleo. 
do insertarse en el nuevo ciclo industrial. Tal es el caso de Asia Oriental, basado en la conversión exportadora hacia la nueva industria electrónica (hardware) seguido por México — aunque bastante más atrás - y los países mencionados de Europa Oriental (República Checa, Hungría y Polonia) y Europa Occidental (tal es el caso de Finlandia, que destacará como líder de la telefonía inalámbrica (Dabat y Toledo, 1999). Sin embargo, regiones como el mundo árabe y sobre todo África se distancian de esta integración por sus límites y atrasos tecnológicos y económicos.

En cuanto a la industria del software y servicios informáticos, si bien Estados Unidos es el principal productor y consumidor a nivel global, ${ }^{8}$ países como Irlanda, India e Israel han alcanzado crecimiento e inserción en los mercados internacionales. Fuera de estos tres casos, los más estudiados y difundidos a nivel internacional, hay otras experiencias de ingreso tardío pero que también demuestran un cierto dinamismo en esa industria. Tal es el caso de Taiwán, China, Singapur, Tailandia, Corea, Malasia, Filipinas y Vietnam. También están teniendo un desarrollo interesante, aunque claramente mucho más acotado que India, Irlanda e Israel, países latinoamericanos como Brasil, Argentina, Uruguay, Costa Rica y México.

Cabe preguntarnos, ¿por qué algunos países y regiones periféricas han logrado insertarse en el nuevo ciclo industrial mundial y otras no? Algunos autores (Dabat; Ordóñez, 2003) consideran una de sus principales causas la llamada nueva división internacional del trabajo, en la nueva fase denominada por Gereffi (1995) "división global del trabajo", la cual redefinió las relaciones entre países y regiones del mundo.

Los grandes cambios derivados de la globalización (procesos que unificaron el mercado mundial, liberación comercial, papel de las comunicaciones, reducciones de los costos de transporte) dieron como resultado la homologación a nivel mundial de las enormes desigualdades de costos internacionales de los factores de la producción (fuerza de trabajo, recursos naturales, renta del suelo, costos de transporte, acceso al capital, entre otros).

$8 \quad$ El mercado mundial de Ti alcanzó en 2001 alrededor de los 1.4 billones de dólares. Mientras que el mercado de software llegó a 196200 millones de dólares, el de servicios informáticos alcanzó los 425700 millones de dólares. El crecimiento y su consecuente expansión del sector de TI ha sido vertiginoso en años recientes, con tasas de crecimiento del orden del 6.9\% anual entre 1993 y 2001 , correspondiéndole al software un crecimiento anual de $13.4 \%$ y a los servicios informáticos de $10.2 \%$ anual. El mercado mundial de Tecnologías de la Información se encuentra concentrado en los países más industrializados, en particular en los Estados Unidos (40\% del mercado mundial de TI, $49 \%$ del mercado de software y $47 \%$ de servicios informáticos en 2001). En este panorama internacional, algunos países de Asia (India, China, Corea, Taiwán, Hong Kong) y en menor medida algunos países de América Latina (Brasil, Argentina) participan con 5 a 6\% del mercado global de TI y servicios informáticos. México se ubica en el puesto número 19 en esta clasificación con alrededor de $0.6 \%$ del mercado mundial, así como con $0.3 \%$ en software y $0.4 \%$ en servicios informáticos (hasta el año 2001). Fuente: WITSA (2000 2002). 
Otro elemento importante es la posibilidad de empresas y países de desarrollar procesos manufactureros en los diversos niveles de la cadena de valor, y estrategias propias de comercialización para los productos resultantes.

La nueva división internacional del trabajo, ${ }^{9}$ en la conformación del nuevo ciclo industrial dirigido por el SE-I, permitirá aún más:

a) la incorporación al comercio internacional de un conjunto de nuevos procesos productivos y productos intensivos en trabajos semicalificados y calificado (técnico, ingenieril y aún científico), en lugar del trabajo exclusivamente descalificado de las primeras industrias incorporadas a la nueva división internacional del trabajo como confección, calzado y juguete) y $b$ ) las acentuadamente mayores posibilidades de aprendizaje tecnológico de empresas y países de los diferentes segmento de las SE-I, en relación con los sectores productivos, casi únicamente intensivos en trabajo (Idem, 2003:58).

De acuerdo con lo dicho, encontramos la conformación de nuevos eslabonamientos productivos de la cadena de valor en los países de industrialización tardía que pueden desarrollar los procesos manufactureros y de servicios, objeto de una división interindustrial del trabajo o de relocalización internacional (desarrollo de la una red productiva internacional).

Es en este escenario donde los países en vías de industrialización pueden incorporar eslabones productivos del SE-I, caracterizados por esferas laborales más calificadas, algunos de ellos intensivos en conocimiento como el diseño de productos o de software.

Ahora bien, la inserción de estos países de industrialización tardía o en vías de industrialización en el nuevo ciclo industrial depende de las posibilidades que les confieran sus capacidades socioeconómicas, empresariales, educacionales, infraestructura y la existencia de políticas industriales activas coherentes con tal finalidad. En algunos países mencionados como exitosos, los gobiernos ya han apoyado el desarrollo de la industria, por su carácter intensivo en tecnología y recursos humanos calificados.

\section{Experiencias de India, Irlanda e Israel}

Para nuestra investigación han sido analizados los casos de países más exitosos, tales como India, Irlanda e Israel, así como países de América Latina: Brasil, Argentina, Uruguay y Costa Rica. Para llevarla a cabo se tomaron como referencia estudios realizados en estos países. ${ }^{10}$

Podemos identificar su desarrollo respecto a la industria del software identificando modelos diferentes en cuanto a: a) el involucramiento del Estado en el proceso de desarro-

9 Este concepto fue utilizado inicialmente en relación con la tendencia surgida en la segunda mitad de los sesenta, de crecimiento de las exportaciones de productos manufactureros intensivos en trabajo desde los países en desarrollo a los países industriales, a partir de un proceso de relocalización del capital hacia los países periféricos de trabajo descalificado barato (Frobel, Heinrichs y Kreyre, citados por Dabat, Ordóñez, 2003).

10 D. Hoch, C. Roeding, G. Purkert y S. Lindner, Secrets of Software Success. Managements Insights from 100 Software Firms around the World, Harvard Business School Press, Boston, 1999; Nasscom 
llo del sector (políticas públicas activas en el ámbito impositivo, normativo, de infraestructura, educativo, entre otros); $b$ ) en cuanto a la orientación de su producción y al tipo predominante de actividades; $c$ ) la orientación de mercado elegida; $d$ ) facturación, número de empresas y de empleados que lideran la expansión del sector.

India, Irlanda e Israel han tenido como detonante del crecimiento sus exportaciones. En América Latina, el cuadro se muestra más complejo, ya que tanto Brasil como Argentina han centrado su desarrollo en el mercado interno, iniciando en forma parcial y reciente el proceso exportador. En Uruguay y Costa Rica la industria ha surgido más vinculada a las exportaciones por las debilidades de su propio mercado interno.

En los primeros tres casos han existido estrategias sustentadas por instituciones públicas, producto de la participación del gobierno con políticas industrial y educativa activas, ofreciendo infraestructura apta (transporte y servicios en general, estímulo al asociacionismo empresarial, facilitación de tareas burocráticas) así como ventajas impositivas y aduaneras que permitieron desarrollar al sector, en particular sus exportaciones. Si bien India e Israel ya contaban con recursos humanos calificados, el gobierno incentivó la formación de personal de alto nivel de calificación en instituciones de excelencia. Este es uno de los factores de mayor peso en el ingreso de estos países en los mercados de exportación. Cabe destacar el dominio del idioma inglés. Contemporáneamente se ha estimulado la oferta de capital de trabajo y de riesgo a través de subsidios, préstamos preferenciales y exenciones, entre otros. En Israel, por ejemplo, el desarrollo de capital de riesgo tuvo un papel destacado. Asimismo, estos tres países han invertido en I\&D de manera importante, aunque no todos con los mismos resultados: mientras que ha sido clave en Israel, en India han sido menos exitosos.

En América Latina, los esfuerzos del gobierno han sido débiles y dispersos. No existen grandes incentivos fiscales, falta coordinación interinstitucional, y la mayoría de los países todavía no cuenta con un cuadro normativo adecuado sobre la materia ni con fuentes de capital de riesgo. Por lo general, todos cuentan con buena infraestructura en telecomunicaciones. Destaca la calidad de los recursos humanos, si bien la disponibilidad de profesionales de máximo nivel para la industria es aún escaso. A diferencia de las otras experiencias exitosas, no cuentan con recursos para el ámbito de la I\&D.

(2000), "Indian IT Software and Services Industry", disponible en http://www.nasscom.org; (2001a), "Indian IT Software and Services Industry", disponible en http://www.nasscom.org; (2001b), "Indian Iт Software and Services Industry", disponible en http://www.nasscom.org; Enterprise Ireland's Nationatl Informatics directory para Irlanda; Israel Association of Software House para Israel; Instituto de Estudios Económicos en Software y Ciencia y Tecnología y Proyecto MIT-SOFTEX -W -class (2002) para Brasil; Chudnovsky, Daniel, et al. (2001), El sector de software y servicios informáticos (SSI) en la Argentina: Situación actual y perspectivas de desarrollo, mimeo, para Argentina; Ministerio de Industria, Energía y Minería, Uruguay (1999). Informe Sector Software y Stolovich et al. (2001) para Uruguay, Caprosoft para Costa Rica. 
En cuanto a estándares de calidad tipo $\mathrm{CMM}^{11}$ e ISO, ${ }^{12}$ sobresale la gran cantidad de empresas de India que han calificado con el modelo смм en los niveles cuatro (41 empresas) y cinco (62 empresas). Irlanda, por su parte, cuenta con una sola empresa con tal certificación (nivel cuatro), lo cual indica que este país no le da importancia. Israel tampoco sobresale por tenerlas, ya que cuenta solamente con una empresa de nivel cuatro (fuente: Software Engineering Institute).

Entre los países de industrialización tardía destaca India por sus exportaciones. Más de $75 \%$ de los ingresos totales generados por el sector en 2002 , fueron por concepto de exportaciones, con un ingreso de 7800 millones de dólares. En cuanto al tipo de actividades predominante, muchas empresas se dedicaron a ofrecer servicios de desarrollo y mantenimiento, realizados por un grupo de programadores que se trasladaban al domicilio del cliente . Por lo tanto, la actividad de software orientado exclusivamente a la exportación se concentró en el rubro de "servicios profesionales". El destino fundamental de éstos es Estados Unidos (58\%), sigue Europa (principalmente Reino Unido) con 21\% y Japón y otros países del sudeste asiático con alrededor de 4\%. En los últimos años, el sector vinculado con las exportaciones ha adoptado actividades más complejas como offshore y outsourcing. ${ }^{13}$ Irlanda exportó, en 2002, 8500 millones de dólares y la composición se diferencia del modelo hindú por el predominio de software empaquetado. La Unión Europea, y en particular el Reino Unido, reúne 70\% de las exportaciones irlandesas. Por otra parte, Irlanda no parece haberse transformado en un centro importante de programación offshore como la India. La industria israelí de software alcanzó por concepto de exportaciones en 2001 un valor estimado en 3000 millones de dólares y ha tenido un desempeño destacado en áreas de seguridad y tecnologías antivirus. Muchas empresas se dedican a desarrollar software empaquetado con cierto nivel de sofisticación para mercados extranjeros. Los destinos de Israel están más diversificados que los de otros países: a Estados Unidos va $38 \%$ y $37 \%$ van dirigidas a Europa Occidental.

Los grandes países de América Latina - Brasil y Argentina - no alcanzan todavía una inserción exitosa en el mercado mundial. El modelo que configuran es el de producción para el mercado interno. En Brasil, las exportaciones alcanzaron en 2001 cifras por 84.1 millones de dólares. Si bien abastece al mercado interno, las empresas enfocan más su producción en áreas de mercado competitivas mundialmente, tales como el segmento ban-

11 Se trata de un marco de trabajo que permite establecer, mejorar y medir la madurez de los procesos de producción (Capability Maturity Model).

12 La norma Iso (Internacional Standars Organization) especifica los requisitos de un sistema de gestión de calidad que puede utilizarse para su aplicación interna en las empresas, para certificación con fines contractuales.

13 Software de servicios a distancia (offshore outsourcing): administración remota de aplicaciones, desarrollo y mantenimiento de aplicaciones y, en fase más avanzada, administración integral de todo el departamento de sistemas de una empresa. 
cario, las telecomunicaciones e infraestructura energética. Otra característica importante de la producción en Brasil es el mercado de software a la medida y software embebido, habiendo alcanzado una importante madurez tecnológica para el mercado nacional y para abrirse a las exportaciones. También es importante el desarrollo de software en comercio electrónico y en prestación de servicios. Todas estas actividades ponen de manifiesto el esfuerzo que están realizando en Brasil las empresas por crear software competitivo e innovador. Exportó 35 millones de dólares en 2000. En este país la mayor parte de las empresas se dedican a la prestación de servicios (absorben casi la mitad del empleo y facturación sectorial), seguida de un pequeño grupo de empresas cuya actividad principal es comercializar software producido en el extranjero. Por último se destacan las empresas que lo desarrollan orientado al mercado interno. Uruguay y Costa Rica, en cambio, cuentan ya con un importante potencial para la exportación. En Uruguay las exportaciones alcanzaron en 2000 un ingreso de 79.4 millones de dólares y en Costa Rica de alrededor de 50 millones de dólares. Las empresas uruguayas que lo producen se han especializado en ciertos nichos alcanzando la excelencia y exportando productos para pequeñas farmacias y casas de cambio. El principal destino de sus exportaciones es América del Sur que representa 64.3\%. En Costa Rica se ha verificado un gran crecimiento en la producción y exportación de software, primero hacia economías vecinas y, más recientemente, a Europa, Asia e incluso África. Más de 70\% de las empresas locales lo exportan a estos países en áreas como bancos, finanzas, recursos humanos, turismo e inteligencia artificial, entre otros.

En India los ingresos del sector del software y servicios informáticos alcanzaron en 2002, 10200 millones de dólares. El número de empresas que conforman el sector es de 6 000. La industria genera un total de 400000 empleados. En Irlanda, datos de 2002 indican que la industria alcanzó una facturación por 10000 millones de dólares y está constituida por 900 firmas del sector de software y servicios informáticos que emplean a unos 30000 trabajadores. Israel factura 4100 millones de dólares y cuenta con 400 empresas de software que emplean unas 15000 personas. Entre los países de América Latina que se tomaron en cuenta para nuestro estudio, Argentina facturó alrededor de 2000 millones de dólares en 2000. El sector está conformado por unas 500 empresas de software y servicios informáticos y emplea unas 15000 personas. Brasil comercializó en 20013883 millones de dólares. Cuenta con 2398 empresas desarrolladoras de software (no incluye los servicios informáticos) y empleó para todo el sector de 121000 a 158000 personas. Uruguay facturó un total de 240 millones en 2000, cuenta con 250 empresas que se dedican al desarrollo de software y con el sector de servicios informáticos emplea un total de 2500 a 3000 personas. Costa Rica si bien no pudimos recabar datos de su facturación, sabemos que cuenta con 150 empresas que dedican a producción y desarrollo de software y emplea unas 3500 personas. 
Si bien las experiencias más estudiadas para esta investigación —-India, Irlanda e Israel— aparecen como las más exitosas a nivel internacional, no están por ello exentas de factores que impiden su pleno desarrollo. En el caso de India, la industria en conjunto presenta poca capacidad innovativa, restándole al sector mayor dinamismo en un futuro cercano (D'Costa, 2000). El otro factor de incertidumbre que presenta este país es la competencia creciente de otras realidades respecto al costo de la mano de obra calificada. Hay países que reportan costos salariales mucho más bajos que los vigentes en India; tal es el caso de Vietnam o países europeos como Rusia y Rumania.

En el caso de Irlanda podemos señalar que: $a$ ) hay escaso desarrollo de actividades de I\&D; b) las empresas especializadas en servicios enfrentan la competencia creciente de países con salarios más bajos; $c$ ) el número de graduados en informática es insuficiente en función de la demanda pronosticada, y se mencionan ciertas carencias en su formación; $d$ ) hay escaso énfasis en la formación de posgrado; $e$ ) las firmas locales exhiben debilidades en materia de distribución, capacidad de management y financiamiento; $f$ ) también hay deficiencias serias en el área de ventas y marketing (estudio realizado por Price Waterhouse, 1999, citado por López, 2003).

Fuera de estos tres casos, que son los más estudiados y difundidos a nivel internacional, hay algunas otras experiencias de ingreso tardío, como mencionamos anteriormente, que también demuestran cierto dinamismo en la industria del software y servicios informáticos. Tal es el caso de Taiwán, China, Singapur, Tailandia, Corea, Malasia, Filipinas y Vietnam. Otros países con desarrollo relativamente menor están también adquiriendo relevancia en el sector. Corea, si bien facturó alrededor de 700 millones de dólares anuales, sus exportaciones son muy bajas y están mayoritariamente dirigidas a Estados Unidos y Japón. Singapur, a partir de la decisión de Microsoft de centralizar la manufactura y distribución para el sudeste asiático tendrá una repercusión importante en el desarrollo de la industria. Las ventas del sector alcanzaron en 1997, 1660 millones de dólares, y 30\% fueron de exportación. China comenzó a desarrollarse alrededor de los años ochenta, y hoy cuenta con 5000 firmas y una facturación de 9600 millones de dólares.

\section{La industria del software en México}

En México, la industria del software atraviesa también un proceso de maduración incipiente que se manifiesta en un crecimiento casi ininterrumpido durante los años recientes. El total de facturación de software en paquete llegó en 2002 a 676.78 millones de dólares y la del software a la medida, alcanzó en la misma fecha 160.51 millones de dólares. Podemos agregar que por concepto de exportaciones se facturaron 121.89 millones de dólares. Asimismo, cabe señalar que $94 \%$ del software empaquetado es de origen extranjero. Se calcula que forman parte del sector unas 250 empresas. 
Las compañías que lo desarrollan en México son pocas y en su mayoría son micro y pequeñas empresas. Una de las causas identificadas en nuestra investigación que impide el surgimiento de nuevas firmas es la cantidad de software cautivo producido por sectores no especializados tales como educación, gobierno federal, estatal, empresas de mercado (manufactura, distribución, finanzas, procesos y servicios). El total de facturación de este sector es de 751.14 millones, cifras muy superiores al total facturado por software a la medida - desarrollado en México por las empresas especializadas del sector-que es de 160.51 millones. Representa 4.67 veces más.

Las empresas en México se encuentran concentradas en áreas específicas, con asentamientos en ciudades. ${ }^{14}$ Configuran modelos híbridos poco ajustables dentro de los modelos de clusters o tecnopolos.

Los casos analizados para nuestro estudio focalizaron a 30 empresas desarrolladoras de software a la medida, nacionales e internacionales radicadas en México. ${ }^{15}$ Con base en los datos recogidos, se obtuvieron los siguientes resultados:

Las empresas encuestadas son en su mayoría relativamente jóvenes. Si bien algunas de ellas ya desarrollaban software en la década de los ochenta, el boom se concentra en la década de los noventa y continúa hasta nuestros días.

Estas 30 empresas están compuestas en su mayoría, si las medimos por número de empleados, en 13 pequeñas y 8 micro, y de acuerdo a su facturación sobresalen 14 micro, 6 medianas y 6 corporativas. Estos datos ponen de manifiesto altos niveles de producción concentrados en muy pocas empresas.

El total de facturación de las firmas encuestadas asciende a 236.65 millones de dólares, lo cual representa $71 \%$ del total del mercado de software a la medida. Prácticamente en ellas se concentra la mayor parte de la producción del país. Si bien el sector aparece orientado al mercado interno, por la cantidad de empresas, las exportaciones arrojan una facturación de 121.89 millones de dólares, lo cual demuestra una dualidad entre la producción

14 La mayor concentración se encuentra en el Distrito Federal (55\%), seguida de Monterrey, Guadalajara y Puebla.

15 Se aplicó una encuesta y entrevistas a profundidad a 30 empresas desarrolladoras de software a la medida, nacionales e internacionales radicadas en México: Adam Technologies; CD Media soluciones; Certum; Consultores Profesionales; Ddemesis; Desarrollo en Sistemas de Informática; Desarrollos Computacionales de México; Dipros; EDs; Esiglo; GGS; Grupo Asercom; Grupo Empresarial Optima; Grupo Jade; Grupo Matriz; Hildebrando; IBM México; Internacional de Sistemas; Intra Portales; Kernel Corporativo; Kiven; Netropology; Northware; Pacific Sofá; Praxis; Prisma Computación; Sistemas Administrativos Computarizados; Softtek; Tecnología Esencial; Ultrasist.

En esta fase se contó con la colaboración de la empresa Select, IDC, que ayudó en la búsqueda, recolección y sistematización de datos. Cabe señalar que, a pesar de los esfuerzos realizados, no fue posible estudiar todo el universo de empresas (unas 250), porque el subsector todavía tiene un carácter informal. Por tanto, se consideró oportuna y representativa la muestra de estas 30 empresas, debido a su competitividad e importancia en el ámbito de la producción y exportación. Estas empresas constituyen $70 \%$ del facturado de software a la medida en México y representan la parte más destacada de su industria en México. 
interna y la producción para la exportación. Esto obedece a la concentración de la producción para exportar en sólo tres grandes firmas: IBM, Softtek y Ddemesis; y a la gran producción de software cautivo por sectores no especializados, mencionados anteriormente, quienes compran poco software en el mercado. Estos factores contribuyen a frenar el desarrollo de un mercado nacional que facilitaría a las empresas adquirir escala.

El personal ocupado en desarrollo de software (considerados como técnicos), en las compañías encuestadas llega a 3814.7 y el personal administrativo a 468. Las empresas cuentan con egresados de licenciaturas, pero con poco personal con maestrías y una ausencia casi absoluta de profesionales con doctorado. Los empresarios lamentan el tiempo que tienen que dedicar a la formación dentro de sus propias empresas.

Considerando la actividad principal de las firmas, se observa que el grupo más importante comprende al sector de manufactura $(26.8 \%)$, aunque también se encontró una alta orientación hacia empresas de informática y telecomunicaciones (19.9\%) y seguros y servicios financieros en $19.8 \%$.

En las empresas encuestadas, los lenguajes tradicionales (Cobol, Pascal, Fortran, Basic, C) son muy pocos utilizados: sólo por 2.6\%. Lenguajes como C\#, Java, Java Script, HTML/ XML se utiliza en $35.6 \%$ de los casos. Oracle, Developer, Power Builder y Visual Basic en 29.8\%. El dominio de lenguajes de última generación permite desarrollar aplicaciones más sofisticadas. La elección de lenguaje estará siempre condicionada a los requerimientos de las aplicaciones por desarrollar así como la destreza de los programadores. En la medida en que el software desarrollado en México es de mediana complejidad, sólo las firmas grandes dominan lenguajes más avanzados.

Los resultados de nuestra investigación muestran que en México existe un claro predominio de la plataforma Windows en sus recientes versiones NT/2000, con 35\% del total de las empresas encuestadas. También es alto el porcentaje de empresas que utilizan el sistema operativo Unix para el desarrollo de software (24.1\%). Con sus distintas versiones, Linux representa $6.2 \%$ del total. El incremento de su uso en algunos sectores puede resultar un factor de competencia, abrir más espacios para generar desarrollos innovativos y abaratar dichos procesos. Aunque su uso todavía es muy bajo, comienza a ser considerado por algunas áreas de gobierno y de mercado.

Los niveles de piratería en México son altos. Según los resultados arrojados por el estudio más reciente (el sexto que se realiza) de la Business Software Alliance (BSA), ${ }^{16}$ efectuado por la International Planning and Research Corp. (IPR), las pérdidas por piratería aumentaron de 133964 millones de dólares en 1999 a 180164 millones de dólares durante 2000. El estudio señala que México y Brasil, las dos economías más grandes de la

16 La Business Software Alliance ( $w w w . b s a . o r g)$ es el organismo dedicado a promover un mundo interconectado seguro y legal. La BSA es la voz de las industrias de software, hardware e Internet del planeta ante los gobiernos y consumidores dentro de un mercado internacional. 
región, tuvieron un porcentaje de piratería constante, entre 1999 y 2000. Sus porcentajes actuales son $56 \%$ y $58 \%$, respectivamente. La tercera economía, Argentina, también se mantuvo en $58 \%$, y Chile, una vez más, presentó el porcentaje más bajo de América Latina: $49 \%$.

El problema en México radica en la inadecuación de la legislación vigente. ${ }^{17}$

Las políticas públicas tendientes a estimular y facilitar el desarrollo de empresas de software competidoras en los planos nacional e internacional, están bien orientadas pero su implantación es muy reciente, por lo que resulta difícil realizar juicios al respecto. En la actualidad, el Programa para el Desarrollo de la Industria de Software (PDIS) es uno de los medios que el ejecutivo federal utiliza para definir las líneas de acción con que llevará a la práctica esa estrategia del PND, mediante la Subsecretaría de Comercio Interior de la Secretaría de Economía. Asimismo, es una de las iniciativas surgidas en el seno del proyecto e-economía, que junto con e-educación, e-salud y e-gobierno integran el proyecto nacional e-México. Con estos datos se puede concluir que las condiciones en México son favorables para que la industria del software siga creciendo. Esto dependerá de que la economía en su conjunto no sufra grande reveses, así como del aumento de la demanda interna mediante la apertura de sectores cautivos, para permitir la generación y desarrollo de más empresas en el territorio.

En cualquier caso, está claro que México necesita mejorar sus ventajas competitivas para lograr los objetivos propuestos. El subsector del software en el país todavía está lejos de alcanzar la madurez organizativa y tecnológica, a la vez que su mercado aún se encuentra en proceso de redefinición, tanto en el ámbito de las exportaciones como del mercado interno.

\section{Consideraciones finales}

A continuación, y a modo conclusivo, presentamos algunos aspectos encontrados en los casos estudiados como respuesta a los interrogantes planteados.

El grado de desarrollo de la industria de software a nivel internacional, con la introducción de las tecnologías de la información y la comunicación en prácticamente todos los sectores productores de bienes y servicios, permite sustentar que se trata de un sector estratégico, de constante crecimiento, cuya competitividad permite a algunas naciones de industrialización reciente su inserción en el mercado internacional.

17 Con la Ley Federal del Derecho de Autor en México se pretenden subsanar los daños que ocasiona la piratería, mediante cobros a los productores de equipos que facilitan a individuos o empresas la re producción de materiales protegidos. El Instituto Mexicano de la Propiedad Intelectual es el órgano encargado de aplicar el derecho de autor. No obstante, la legislación aún carece de los mecanismos suficientes para enfrentar este problema y se tendría que reglamentar sobre la materia de confor midad con criterios y prácticas internacionales, dado que este asunto rebasa las fronteras del país. 
Debemos señalar que todos los países estudiados deberán continuar desarrollando la capacidad innovativa de sus empresas para seguir siendo competitivos, pues, para hacerlo en el nivel internacional, y en menor escala en el local, no es suficiente desarrollar software de aplicaciones a la medida, productos con cierto grado de estandarización o adaptados a los requerimientos particulares del usuario.

La capacidad innovativa de las empresas, así como la especialización en mercados y segmentos más complejos y específicos (como offshore y outsourcing o software para Internet), son los cambios estratégicos a los cuales las empresas del sector en los distintos países estudiados deberán atenerse para su desempeño futuro.

Las ventajas en los costos de mano de obra y precios de ventas, que favorecen a algunos países por su situación cambiaria, resultan insuficientes no sólo para alcanzar una inserción exportadora sostenible sino para lograr una mejor posición en sus propios mercados internos.

Pocos países, a excepción de India, implementan programas de calidad, basados en estándares internacionales. En América Latina es casi nula la presencia de desarrollos innovativos de más amplio alcance (salvo algunas experiencias exitosas en Brasil y Costa Rica). La escala relativamente pequeña del mercado interno, en la mayoría de los países de América Latina, dificulta que las firmas locales dediquen esfuerzos significativos para actividades de I\&D.

Otro factor de competitividad importante es el activo con el cual cuentan los países a partir de la disponibilidad de capital humano calificado. Paradójicamente, los países estudiados de América Latina cuentan con recursos humanos en cantidad suficiente pero de calidad insuficiente: faltan profesionales de alto nivel, lo cual limita las posibilidades de avanzar en desarrollos innovativos.

En cuanto al desarrollo de una industria competitiva del software en México pudimos constatar que es factible a partir de la existencia de: a) un conjunto de firmas con una trayectoria importante que operan en el sector tanto a nivel internacional como nacional; b) la ubicación geográfica y cercanía con el mayor mercado del mundo, Estados Unidos, y la presencia de un mercado hispano parlante en él; y c) la existencia de un sistema científico-educativo en desarrollo, dotado de un incipiente sector especializado en electrónica y software.

Dentro de este contexto general, el mercado interno constituye una base económica de grandes posibilidades de desarrollo (un área de oportunidad), no sólo por el aún bajo nivel nacional de consumo de software y las grandes necesidades de incrementarlo, sino porque la mayor parte que es consumido por los grandes usuarios (grandes empresas y entidades públicas) es producido al interior de las mismas, a diferencia, por ejemplo, de lo que ocurre en Brasil, donde la industria especializada abastece a la mayor parte del consumo nacional. Por esa razón, el impulso a su producción en México debe comenzar por el mer- 
cado interno, a partir del papel promotor del Estado, no sólo en el nivel general (asimilación de experiencias exitosas de los países en desarrollo) sino específicamente, como usuariocomprador fundamental, para estimular a las firmas locales a elevar los volúmenes y calidad de su producción, incluyendo a las pequeñas y medianas empresas (las actuales licitaciones están diseñadas para que sólo accedan a ellas firmas de gran tamaño). Para ello se hace necesario modificar la forma en que se hacen las compras del gobierno, como paquetes completos que incluyen software, hardware, servicio, entre otros, contribuyendo aún más a favorecer a las grandes compañías.

Pero lo expuesto no basta de ninguna manera para desarrollar una industria avanzada. Para ello es necesario combinar ese primer objetivo con, por lo menos, otros tres:

a) El desarrollo del software de exportación, aprovechando la presencia en el país de fuerza de trabajo calificada de bajo costo y muy buen nivel potencial de competitividad internacional. Este tipo de actividad la realizan unas pocas empresas trasnacionales, pero a una escala menor que las de la industria electrónica de hardware. Para este desarrollo es necesario estudiar cuidadosamente la experiencia exitosa de los países considerados en este trabajo, y alentar la construcción de cadenas nacionales de proveedores y, eventualmente, de empresas exportadoras nacionales.

b) La constitución de un fuerte sector científico-educativo tanto a nivel de la formación y la investigación básica, como de su aplicación a la producción. Para ello se necesita ampliar considerablemente la cantidad y calidad de la oferta universitaria de conocimientos (ingenieros de computación, programadores y demás trabajadores especializados requeridos por la industria), así como la relación entre universidad y empresa.

c) El impulso a la conformación de polos de concentración regional de la producción y al conocimiento del tipo de los existentes en India. Esto resulta fundamental para superar la actual dispersión geográfica y falta de articulación funcional de la industria (desarrollo de una división articulada del trabajo a partir de la constitución de cadenas productivas).

En síntesis, México necesita desarrollar simultáneamente su mercado interno (prioridad inmediata) así como posicionarse en el nivel internacional. Para que este cambio sea posible se requiere de iniciativas públicas (promotoras y científico-educativas) y privadas dirigidas específicamente a tal objetivo. La compleja situación del sector no invalida esta posibilidad, sino que la hace más necesaria. 


\section{Bibliografía}

Chudnovsky, Daniel, et al., El sector de software y servicios informáticos (SSI) en la Argentina: Situación actual y perspectivas de desarrollo, mimeo, 2001.

Dabat Alejandro, "Globalización, Capitalismo actual y nuevas configuración espacial del mundo", en Basave Jorge y otros (coordinadores) Globalización y alternativas incluyentes para el siglo XXI, CRIM-UnAM, M.A. Porrúa, México, 2002.

y Ordoñez, Sergio, Revolución Informática.

Nuevo ciclo industrial y división internacional del trabajo: marco general para el estudio de la inserción internacional de México. CRIM-IIEDUNAM. En prensa. México, 2003.

D'Costa, A.P., Export Growth and Path-Dependencie The Locking-in of Innovations in The Software Industry. tTe, International Conference on Technology Policy and Innovation, Curitiba. Agosto, 2000.

Formenti, Carlo, Mercanti di Futuro, Einaudi, Turín, 2002.

Gereffi, G., "Global production Systems and Third Word Development", en B. Stalling (editor), Global Change, Regional Response: The New Internaqtional Context of Development, Nueva York, Cambridge University Press, 1995.

Halal William y Kenneth Taylor, Competing for the future, Harvard Business Scholl Press, 1999.
Hoch, D.; C. Roeding; G. Purkert y S. Lindner, Secrets of Software Success. Managements Insights from 100 Software Firms around the World, Harvard Business School Press, Boston, 1999.

Institute of Technology, A Indústria de Software no Brasil, 2002: Fortalecendo a Economia do Conhecimento, MIT-SOFTEX-w Class. Mimeo, 2003.

López, Andrés, Componente B: Diseño de Análisis de resultados de la segunda encuesta Argentina de innovación 1997/2001, BID-CEPAL, 2003.

Ministerio de Industria, Energía y Minería, Informe Sector Software, Montevideo, junio, 1999.

Miller, Arnold, "Building a Modern Electronic Industry", en Developing the electronics industry. Washington D.C., 1993.

Mochi Alemán, Prudencio, La industria del software en México en el contexto internacional y latinoamericano, tesis de doctorado, Facultad de Ciencias Políticas y Sociales, UnAM, 2003.

Nasscom, "Indian IT Software and Services Industry", disponible en http://www.nasscom.org, 2000.

, "Indian IT Software and Services Industry", disponible en http://www.nasscom.org, 2001a.

Revelli Marco, en Zachary, Pascal, Prefazione in edizione italiana. I guerrieri del software, UTET, 2002, pp. 3-23.

Zubrow, D. et al., "Maturity Questionnaire", en SEI Special Report, cMU/SEI 94-SR-007. 